\title{
Periodic and subharmonic solutions for a class of second-order $p$-Laplacian Hamiltonian systems
}

\author{
Hairong Lian ${ }^{1 *}$, Dongli Wang ${ }^{1}$, Zhanbing Bai ${ }^{2}$ and Ravi P Agarwal ${ }^{3}$
}

"Correspondence: lianhr@126.com

'School of Science, China University of Geosciences, Beijing, 100083,

P.R. China

Full list of author information is available at the end of the article

\begin{abstract}
In this paper, the periodic and subharmonic solutions are investigated for a class of second-order non-autonomous ordinary differential equations with a $p$-Laplacian. With the perturbation technique and the dual least action principle, some existence results are given of solutions to the convex $p$-Laplacian systems.
\end{abstract}

Keywords: $p$-Laplacian; periodic solution; subharmonics; dual least action principle

\section{Introduction}

In this paper, we consider the second-order ordinary differential equations with a $p$ Laplacian

$$
\frac{d}{d t} \Phi_{p}(\dot{x}(t))+\nabla F(t, x(t))=0, \quad \text { a.e. } t \in \mathbb{R},
$$

where $p>1, x=\left(x_{1}, x_{2}, \ldots, x_{N}\right), \Phi_{p}(x)=|x|^{p-2} x$. Here $|\cdot|$ stands the Euclidean norm in $\mathbb{R}^{N}$. In what follows we always suppose that $F: \mathbb{R} \times \mathbb{R}^{N} \rightarrow \mathbb{R},(t, x) \rightarrow F(t, x)$ is measurable and $T$-periodic in $t$ for every $x \in \mathbb{R}^{N}$, continuously differentiable and convex in $x$ for a.e. $t \in \mathbb{R}$. When $p=2$, (1) reduces to the second-order Hamiltonian system

$$
\ddot{x}(t)+\nabla F(t, x(t))=0, \quad \text { a.e. } t \in \mathbb{R} \text {. }
$$

Since $F$ is $T$-periodic in $t$, it is natural to seek $T$-periodic solutions of (1) and (2). Also, $F$ is $k T$-periodic for $k \in \mathbb{N}$, one can search the $k T$-periodic solutions, which is called subharmonic solutions. By a subharmonic solution, it means a $k T$ periodic solution with $k \geq 2$ an integer, that is, the minimal period is strictly greater than $T$. When $k=1$, it is a periodic solution or harmonic. Clearly, a solution $x$ of (1) over $[0, k T]$ verifying $x(0)=x(k T)$ and $\dot{x}(0)=\dot{x}(k T)$ can be extended by $k T$-periodicity over $\mathbb{R}$ to give a $k T$-periodic solution. So, it is fine to study the periodic boundary value of problem of $(1)$ over $[0, k T]$ to present conditions for the existence of the periodic and subharmonic solutions of (1). For the study of the subharmonic solutions, we refer to [1-5] for a few examples.

The dual action principle was firstly introduced by Clarke [6] and developed by Clarke and Ekeland [7-11], which is from the spirit of optimal control theory and convex analy-

\section{首 Springer}

(c) 2014 Lian et al.: licensee Springer. This is an Open Access article distributed under the terms of the Creative Commons Attribution License (http://creativecommons.org/licenses/by/4.0), which permits unrestricted use, distribution, and reproduction in any medium, provided the original work is properly credited. 
sis; see [12]. Following this use and the direct variational method, the periodic solutions are obtained for the Hamiltonian system of (2) in $[2,5,13,14]$ and the references therein. Mawhin and Willem [2] presented the existence results of solutions to the more general systems by using such a principle and the perturbation technique argument. In 2007, Tian and Ge [15] generalized the existence results to the $p$-Laplacian system (1). By transforming the variable, they found a first-order Hamiltonian system equivalent to the secondorder $p$-Laplacian system (1). Then by applying the Clarke duality, the perturbation technique, and the least action principle, they obtained the existence result of the periodic solution. The authors [16] also discussed the multi-point boundary value problem of a second-order differential equation with a $p$-Laplacian.

Motivated by the work listed above, we aim to discuss the periodic and subharmonic solutions to the $p$-Laplacian systems of (1). The proofs are depending on the dual least action principle and the perturbation arguments. To the best of our knowledge, there is no paper discussing the subharmonics solutions of $p$-Laplacian system by using the dual least action principle. This paper is a first try. The existence of the periodic solutions obtained in this paper slightly improves the result in [15]. New arguments are considered for the posterior estimates of the periodic solutions and the existence of subharmonic solutions are presented, which extend those in $[2,5]$.

Other existence results for periodic and subharmonic solutions of the $p$-Laplacian differential equation using other variational methods can be found in [4, 17-24].

The paper is organized as follows. In Section 2, we establish the variational structure of (1) and transfer the existence of the solution into the existence of a critical point of the corresponding functional. The dual action is mainly discussed here. In Section 3, sufficient conditions are presented to guarantee the existence of the periodic solution of (1). We also estimate the prior bounds of all the periodic solutions of (1). The existence of the subharmonic solutions are given in the last section.

\section{Preliminary and dual action}

Let $k \geq 1$ be any integer and $p>1$ a constant. The Sobolev space $W_{k T}^{1, p}\left(0, k T ; \mathbb{R}^{N}\right)$ is the space of functions $x:[0, k T] \rightarrow \mathbb{R}^{N}$ with $x \in L^{p}\left(0, k T ; \mathbb{R}^{N}\right)$ having a weak derivative $\dot{x} \in$ $L^{p}\left(0, k T ; \mathbb{R}^{N}\right)$ and $x(0)=x(k T)$. The norm over $W_{k T}^{1, p}$ is defined by

$$
\|x\|_{W_{k T}^{1, p}}=\left(\int_{0}^{k T}|x(t)|^{p} d t+\int_{0}^{k T}|\dot{x}(t)|^{p} d t\right)^{\frac{1}{p}} .
$$

Here, we recall that

$$
|x|=\sqrt{\sum_{i=1}^{N}\left|x_{i}\right|^{2},} \quad\|x\|_{L^{p}}=\left(\int_{0}^{k T}|x(t)|^{p} d t\right)^{\frac{1}{p}}, \quad\|x\|_{\infty}=\max _{t \in[0, k T]}|x(t)| .
$$

Consider the spaces $X$ and $Y$ defined by

$$
\begin{aligned}
& X=\left\{u=\left(u_{1}, u_{2}\right):[0, k T] \rightarrow \mathbb{R}^{2 N}, u_{1} \in W_{k T}^{1, p}\left(0, k T ; R^{N}\right), u_{2} \in W_{k T}^{1, q}\left(0, k T ; R^{N}\right)\right\}, \\
& Y=\left\{v=\left(v_{1}, v_{2}\right):[0, k T] \rightarrow \mathbb{R}^{2 N}, v_{1} \in W_{k T}^{1, q}\left(0, k T ; R^{N}\right), v_{2} \in W_{k T}^{1, p}\left(0, k T ; R^{N}\right)\right\},
\end{aligned}
$$


with the norm $\|u\|_{X}=\left\|u_{1}\right\|_{W_{k T}^{1, p}}+\left\|u_{2}\right\|_{W_{k T}^{1, q}}$ and $\|v\|_{Y}=\left\|v_{1}\right\|_{W_{k T}^{1, q}}+\left\|v_{2}\right\|_{W_{k T}^{1, p}}$, respectively. Here $q$ is a constant such that $\frac{1}{p}+\frac{1}{q}=1$. It is easy to verify that $X$ and $Y$ are reflexive Banach spaces and $X^{*}=Y$.

For any $h=\left(h_{1}, h_{2}\right) \in L^{1}\left(0, k T ; \mathbb{R}^{N} \times \mathbb{R}^{N}\right)$, the mean value is defined by

$$
\bar{h}=\frac{1}{k T} \int_{0}^{k T} h(t) d t
$$

Similarly, we can define $\bar{h}_{1}$ and $\bar{h}_{2}$. We denote by $\tilde{Y}$ the subspace of $Y$ by

$$
\tilde{Y}=\{v \in Y: \bar{v}=0\} .
$$

We easily find the following inequality.

Lemma 2.1 For every $u_{1} \in W_{k T}^{1, p}$ and $\bar{u}_{1}=0$, we have

$$
\left\|u_{1}\right\|_{L^{p}} \leq k T\left\|\dot{u}_{1}\right\|_{L^{p}}
$$

For every $u_{2} \in W_{k T}^{1, q}$ and $\bar{u}_{2}=0$, we have

$$
\left\|u_{2}\right\|_{L^{q}} \leq k T\left\|\dot{u}_{2}\right\|_{L^{q}}
$$

Let $u_{1}=x, \alpha u_{2}=\Phi_{p}(\dot{x})$, then the second-order $p$-Laplacian system (1) can be changed to the first-order ones,

$$
\left\{\begin{array}{l}
\dot{u}_{1}=\Phi_{q}\left(\alpha u_{2}(t)\right) \\
\dot{u}_{2}=-\frac{1}{\alpha} \nabla F\left(t, u_{1}(t)\right)
\end{array}\right.
$$

where $\alpha>0$ is a parameter. Define $H_{i}:[0, k T] \times \mathbb{R}^{N} \rightarrow \mathbb{R}, i=1,2$, and $H:[0, k T] \times \mathbb{R}^{2 N} \rightarrow$ $\mathbb{R}$ by

$$
\begin{aligned}
& H_{1}\left(t, u_{1}\right)=\frac{1}{\alpha} F\left(t, u_{1}\right), \quad H_{2}\left(t, u_{2}\right)=\frac{\alpha^{q-1}}{q}\left|u_{2}\right|^{q}, \\
& H(t, u)=H_{1}\left(t, u_{1}\right)+H_{2}\left(t, u_{2}\right)
\end{aligned}
$$

for $u=\left(u_{1}, u_{2}\right) \in R^{2 N}$. Then the system (3) can be written by

$$
J \dot{u}(t)+\nabla H(t, u(t))=0,
$$

where $J=\left(\begin{array}{cc}0_{N} & I_{N} \\ -I_{N} & 0_{N}\end{array}\right)$ is the symplectic matrix. Obviously, $J^{2}=-I_{2 N}$ and $(J u, v)=-(u, J v)$ for all $u, v \in \mathbb{R}^{2 N}$.

By the $k T$-periodic property, we have the Hamiltonian action of (4) on $X$ given by

$$
\psi(u)=\int_{0}^{k T}\left(-\frac{1}{2}(J \dot{u}(t), u(t))-H(t, u(t))\right) d t
$$


where $(\cdot, \cdot)$ is the inner product in $\mathbb{R}^{2 N}$. Because the first part of $\psi$ is indefinite, the dual least process is applied to discuss the existence of the periodic solutions of (4).

Let $\Gamma_{0}$ the set of all convex lower semi-continuous functions from $\mathbb{R}^{2 N}$ to $(-\infty, \infty]$ whose effective domain (not equal to $\infty$ ) is nonempty. Then the Fenchel transform $H^{*}(t, \cdot)$ of $H(t, \cdot) \in \Gamma_{0}$ is defined by

$$
H^{*}(t, v)=\sup _{u \in \mathbb{R}^{2 N}}((v, u)-H(t, u))
$$

Similarly, we also can define

$$
F^{*}\left(t, v_{1}\right)=\sup _{x \in \mathbb{R}^{N}}\left(\left(v_{1}, x\right)-F(t, x)\right) .
$$

For more properties of the Fenchel transform, we refer to [2, 12]. By Theorem 2.2 and Proposition 2.4 in [2], we have the following conclusion.

Lemma 2.2 Suppose the following condition holds.

$\left(\mathrm{A}_{0}\right)$ There exist positive constants $\alpha, \delta$, and positive functions $\beta, \gamma \in L^{q}(0, k T ;[0,+\infty))$ such that

$$
\frac{\delta}{p}|x|^{p}-\beta(t) \leq F(t, x) \leq \frac{\alpha}{p}|x|^{p}+\gamma(t)
$$

holds for a.e. $t \in[0, k T]$ and $x \in \mathbb{R}^{N}$.

Then

$$
\frac{1}{q} \alpha^{-\frac{q}{p}}\left|v_{1}\right|^{q}-\gamma(t) \leq F^{*}\left(t, v_{1}\right) \leq \frac{1}{q} \delta^{-\frac{q}{p}}\left|v_{1}\right|^{q}+\beta(t), \quad \text { a.e. } t \in[0, k T] .
$$

Furthermore, we have $F^{*}(t, \cdot) \in C^{1}\left(\mathbb{R}^{N}\right)$ and

$$
\left|\nabla F^{*}\left(t, v_{1}\right)\right| \leq\left(\frac{p}{\delta}\left(\left|v_{1}\right|+\beta(t)+\gamma(t)\right)+1\right)^{q-1}, \quad \text { a.e. } t \in[0, k T] .
$$

From the definition of $\mathrm{H}_{2}$ and Lemma 2.2, we have the following result.

Corollary 2.3 $H_{2}^{*}\left(t, v_{2}\right)=\frac{1}{p \alpha}\left|v_{2}\right|^{p}$.

Easily we find $H^{*}(t, v)=\frac{1}{\alpha} F^{*}\left(t, v_{1}\right)+H_{2}^{*}\left(t, v_{2}\right)$. So when $F$ satisfies the condition $\left(\mathrm{A}_{0}\right)$, $H^{*}(t, v)$ is continuously differentiable in $v$ for a.e. $t \in[0, k T]$ and

$$
H^{*}(t, v)=(v, u)-H(t, u) \quad \Leftrightarrow \quad v=\nabla H(t, u) \quad \Leftrightarrow \quad u=\nabla H^{*}(t, v) .
$$

Let $v=-J u$ and by duality, we have

$$
\begin{aligned}
\psi(u) & =\int_{0}^{k T}\left(-\frac{1}{2}(J \dot{u}(t), u(t))-H(t, u(t))\right) d t \\
& =\int_{0}^{k T}\left(\frac{1}{2}(\dot{v}(t), u(t))-H(t, u(t))\right) d t
\end{aligned}
$$




$$
\begin{aligned}
& =\int_{0}^{k T}\left(-\frac{1}{2}(\dot{v}(t), u(t))+(\dot{v}(t), u(t))-H(t, u(t))\right) d t \\
& =\int_{0}^{k T}\left(\frac{1}{2}(J \dot{v}(t), v(t))+H^{*}(t, \dot{v}(t))\right) d t
\end{aligned}
$$

So the dual action can be defined on $Y$ by

$$
\mathcal{X}(v)=\int_{0}^{k T}\left(\frac{1}{2}(J \dot{v}(t), v(t))+H^{*}(t, \dot{v}(t))\right) d t
$$

$\mathcal{X}$ is the functional we needed since the critical points of $\mathcal{X}$ on $Y$ coincide with the solutions of (4). Because $\mathcal{X}(v+c)=\mathcal{X}(v)$, it suffices to find a critical point in $\tilde{Y}$. Similarly to the discussion of the related lemmas in reference [15], we have the following results.

Lemma 2.4 Suppose the condition $\left(\mathrm{A}_{0}\right)$ holds. Then $\mathcal{X}$ is continuously differential on $\tilde{Y}$. For any $h \in \tilde{Y}$, we have

$$
\left\langle\mathcal{X}^{\prime}(v), h\right\rangle=\int_{0}^{k T}\left(\frac{1}{2}(J \dot{v}(t), h(t))+\left(\nabla H^{*}(t, \dot{v}(t))-\frac{1}{2} J v(t), \dot{h}(t)\right)\right) d t .
$$

Lemma 2.5 Suppose the condition $\left(\mathrm{A}_{0}\right)$ holds. If $v \in \tilde{Y}$ is a critical point of $\mathcal{X}$, then the function $u(t)=\nabla H^{*}(t, \dot{v}(t))$ is the $k T$-periodic solution of $(4)$ in $X$.

Lemma 2.6 For every $u \in X$, we have

$$
\int_{0}^{k T}(J \dot{u}(t), u(t)) d t \geq-\frac{2 k T}{p}\left\|\dot{u}_{1}\right\|_{L^{p}}^{p}-\frac{2 k T}{q}\left\|\dot{u}_{2}\right\|_{L^{q}}^{q}
$$

For every $v \in Y$, we have

$$
\int_{0}^{k T}(J \dot{v}(t), v(t)) d t \geq-\frac{2 k T}{q}\left\|\dot{v}_{1}\right\|_{L^{q}}^{q}-\frac{2 k T}{p}\left\|\dot{v}_{2}\right\|_{L^{p}}^{p} .
$$

Remark 2.1 There are some extended versions of the inequalities in Lemmas 2.1 and 2.6; see $[24,25]$.

\section{Periodic solutions}

In this section, we discuss the $k T$-periodic solution of (4). Here we note that the $H(t, u)$ is $T$-periodic in $t$ for each $u \in \mathbb{R}^{2 N}$.

Theorem 3.1 Suppose the following conditions are satisfied.

$\left(\mathrm{A}_{1}\right)$ There exists $l \in L^{p q}\left(0, k T ; \mathbb{R}^{N}\right)$ such that for all $x \in \mathbb{R}^{N}$ and a.e. $t \in[0, k T]$, one has

$$
F(t, x) \geq\left(l(t), \Phi_{p}(x)\right)
$$

$\left(\mathrm{A}_{2}\right)$ There exists $\alpha \in\left(0,(k T)^{-\max \{p, q\} / q}\right), \gamma \in L^{\max \{p, q\}}\left(0, k T ; \mathbb{R}^{N}\right)$ such that for all $x \in \mathbb{R}^{N}$ and a.e. $t \in[0, k T]$, one has

$$
F(t, x) \leq \frac{\alpha^{2}}{p}|x|^{p}+\gamma(t)
$$


( $\left.\mathrm{A}_{3}\right) \int_{0}^{k T} F(t, x) d t \rightarrow+\infty$ as $|x| \rightarrow+\infty, x \in \mathbb{R}^{N}$.

Then the problem (4) has at least one solution $u=\left(u_{1}, u_{2}\right) \in X$ such that $u_{1}$ is the $k T$ periodic solutions of (1) and

$$
v(t)=-J\left[u(t)-\frac{1}{k T} \int_{0}^{k T} u(s) d s\right]
$$

minimizes the dual action

$$
\mathcal{X}: \tilde{Y} \rightarrow(-\infty, \infty], \quad v \mapsto \int_{0}^{k T}\left(\frac{1}{2}(J \dot{v}(t), v(t))+H^{*}(t, \dot{v}(t))\right) d t
$$

Proof From the discussion in Section 2, we can see that if the problem (4) has one solution $u=\left(u_{1}, u_{2}\right) \in X$, then $u_{1} \in W_{k T}^{1, p}$ is for the $k T$-periodic solutions of (1). Now we need to prove the problem (4) has at least one $k T$-periodic solution in $X$. The proof is divided into three parts.

Step 1: Existence of a solution for the perturbed problem.

Choose $\epsilon_{0}>0$ such that

$$
0<\alpha+\epsilon_{0}<\min \left\{(k T)^{1-p},(k T)^{-1}\right\}
$$

and for any $0<\epsilon<\epsilon_{0}$, let

$$
H_{1 \epsilon}\left(t, u_{1}\right)=H_{1}\left(t, u_{1}\right)+\frac{\epsilon}{p}\left|u_{1}\right|^{p} .
$$

Clearly, $H_{1 \epsilon}\left(t, u_{1}\right)$ is strictly convex and continuously differentiable in $u_{1}$ for a.e. $t \in[0, k T]$. From $\left(\mathrm{A}_{1}\right)$ and $\left(\mathrm{A}_{2}\right)$, we have

$$
-\frac{1}{\alpha}|l(t)|\left|u_{1}\right|^{p-1}+\frac{\epsilon}{p}\left|u_{1}\right|^{p} \leq H_{1 \epsilon}\left(t, u_{1}\right) \leq \frac{\alpha+\epsilon}{p}\left|u_{1}\right|^{p}+\frac{1}{\alpha} \gamma(t) .
$$

Because the function $g(s)=\frac{\epsilon}{2 p} s^{p}-\frac{1}{\alpha}|l(t)| s^{p-1}, s>0$, attains its minimum at $s=2(p-$ 1) $|l(t)| / \alpha \epsilon$, we have

$$
-\frac{1}{\alpha p}\left(\frac{2(p-1)}{\alpha \epsilon}\right)^{p-1}|l(t)|^{p}+\frac{\epsilon}{2 p}\left|u_{1}\right|^{p} \leq H_{1 \epsilon}\left(t, u_{1}\right) \leq \frac{\alpha+\epsilon}{p}\left|u_{1}\right|^{p}+\frac{1}{\alpha} \gamma(t) .
$$

Let $H_{\epsilon}(t, u)=H_{1 \epsilon}\left(t, u_{1}\right)+H_{2}\left(t, u_{2}\right)$. Easily we find $H_{\epsilon}^{*}(t, v)=H_{1 \epsilon}^{*}\left(t, v_{1}\right)+H_{2}^{*}\left(t, v_{2}\right)$, where $v=\left(v_{1}, v_{2}\right) \in Y$. From Lemma 2.4 and Lemma 2.5, we find that the perturbed dual action

$$
\mathcal{X}_{\epsilon}(v)=\int_{0}^{k T}\left(\frac{1}{2}(J \dot{v}(t), v(t))+H_{\epsilon}^{*}(t, \dot{v}(t))\right) d t
$$

is continuously differentiable on $\tilde{Y}$ and if $v_{\epsilon} \in \tilde{Y}$ is a critical point of $\mathcal{X}_{\epsilon}$, the function $u_{\epsilon}$ defined by

$$
u_{\epsilon}(t)=\nabla H_{\epsilon}^{*}\left(t, \dot{v}_{\epsilon}(t)\right)
$$


is a solution of

$$
\left\{\begin{array}{l}
J \dot{u}(t)+\nabla H_{\epsilon}(t, u(t))=0 \\
u(0)=u(k T)
\end{array}\right.
$$

i.e.,

$$
\left\{\begin{array}{l}
\dot{u}_{1 \epsilon}(t)-\Phi_{q}\left(\alpha u_{2 \epsilon}(t)\right)=0 \\
\dot{u}_{2 \epsilon}(t)+\epsilon \Phi_{p}\left(u_{1 \epsilon}(t)\right)+\frac{1}{\alpha} \nabla F\left(t, u_{1 \epsilon}(t)\right)=0 \\
u_{\epsilon}(0)=u_{\epsilon}(k T)
\end{array}\right.
$$

where we rewrite $u_{\epsilon}=\left(u_{1 \epsilon}, u_{2 \epsilon}\right) \in X$. Meanwhile, from Lemma 2.2, we have

$$
H_{\epsilon}^{*}(t, \dot{v}(t)) \geq \frac{1}{q}(\alpha+\epsilon)^{-q / p}\left|\dot{v}_{1}(t)\right|^{q}+\frac{1}{p} \alpha^{-1}\left|\dot{v}_{2}(t)\right|^{p}-\frac{1}{\alpha} \gamma(t)
$$

which together with (10) and Lemma 2.6 implies that

$$
\begin{aligned}
\mathcal{X}_{\epsilon}(v) \geq & \frac{1}{q}\left((\alpha+\epsilon)^{-q / p}-k T\right) \int_{0}^{k T}\left|\dot{v}_{1}(t)\right|^{q} d t \\
& +\frac{1}{p}\left(\alpha^{-1}-k T\right) \int_{0}^{k T}\left|\dot{v}_{2}(t)\right|^{p} d t-\int_{0}^{k T} \frac{1}{\alpha} \gamma(t) d t \\
\geq & \delta_{10}\left\|\dot{v}_{1}\right\|_{L^{q}}^{q}+\delta_{20}\left\|\dot{v}_{2}\right\|_{L^{p}}^{p}-\gamma_{0}
\end{aligned}
$$

with $\delta_{10}>0, \delta_{20}>0$. Thus every minimizing sequence of $\mathcal{X}_{\epsilon}$ on $\tilde{Y}$ is bounded by (13) and Lemma 2.1. From the continuity of $H_{\epsilon}$ and the definition of $H_{\epsilon}^{*}$, we can see that the second term of $\mathcal{X}_{\epsilon}$ is weakly lower semi-continuous on $\tilde{Y}$. Meanwhile the first part of $\mathcal{X}_{\epsilon}$ is weakly continuous. So $\mathcal{X}_{\epsilon}$ is weakly lower semi-continuous. This implies that $\mathcal{X}_{\epsilon}$ has a minimum at some $v_{\epsilon} \in \tilde{Y}$. So (11) has a solution $u_{\epsilon}=\nabla H_{\epsilon}^{*}\left(t, \dot{v}_{\epsilon}(t)\right)$.

Step 2: Estimation of $u_{\epsilon}$.

It follows from a similar discussion to Lemma 2.2 that

$$
\left|\nabla H_{1}\left(t, u_{1}\right)\right| \leq\left(p \alpha^{-\frac{q}{p}}\left(\left|u_{1}\right|+|l(t)|\left|u_{1}\right|^{p-1}+\gamma(t)\right)\right)^{p-1}+1
$$

So for any $u_{1} \in \mathbb{R}^{N}, \nabla H_{1}\left(t, u_{1}\right) \in L^{q}[0, k T]$. Then the function

$$
\bar{H}_{1}: \mathbb{R}^{N} \rightarrow \mathbb{R}, \quad u_{1} \rightarrow \int_{0}^{k T} H_{1}\left(t, u_{1}\right) d t
$$

is continuously differentiable. By $\left(\mathrm{A}_{3}\right), \bar{H}_{1}$ has a minimum at some point $\bar{u}_{1} \in \mathbb{R}^{N}$ such that

$$
\int_{0}^{k T} \nabla H_{1}\left(t, \bar{u}_{1}\right) d t=0
$$

Easily, $\nabla H_{2}\left(t, u_{2}\right)=\Phi_{p}\left(\alpha u_{2}\right)$ and

$$
\int_{0}^{k T} \nabla H_{2}(t, 0) d t=0
$$


So, let $\bar{u}=\left(\bar{u}_{1}, 0\right) \in \mathbb{R}^{2 N}$, and then $\dot{v}(t)=\nabla H(t, \bar{u})$ has a unique solution $\omega \in \tilde{Y}$. By duality, we also have

$$
H^{*}(t, \dot{\omega})=(\dot{\omega}, \bar{u})-H(t, \bar{u})
$$

and $H^{*}(\cdot, \dot{\omega}(\cdot)) \in L^{1}[0, k T]$. From the inequality $H(t, \bar{u}) \leq H_{\epsilon}(t, \bar{u})$, we have $H_{\epsilon}^{*}(t, \dot{\omega}) \leq$ $H^{*}(t, \dot{\omega})$, and from (13), we obtain

$$
\delta_{10}\left\|\dot{v}_{1 \epsilon}\right\|_{L^{q}}^{q}+\delta_{20}\left\|\dot{v}_{2 \epsilon}\right\|_{L^{p}}^{p}-\gamma_{0} \leq \mathcal{X}_{\epsilon}\left(v_{\epsilon}\right) \leq \mathcal{X}_{\epsilon}(\omega) \leq \mathcal{X}(\omega)=c_{1}<+\infty
$$

By Lemma 2.1, we have $\left\|v_{\epsilon}\right\|_{Y} \leq c_{2}$, where $c_{1}$ and $c_{2}$ are constants independent of $\epsilon$, as well as the following constants $c_{i}, i=3,4,5$. Furthermore, from $\dot{u}_{\epsilon}=J \dot{v}_{\epsilon}$, we have

$$
\left\|\dot{u}_{1 \epsilon}\right\|_{L^{p}}+\left\|\dot{u}_{2 \epsilon}\right\|_{L^{q}}=\left\|\dot{v}_{1 \epsilon}\right\|_{L^{q}}+\left\|\dot{v}_{2 \epsilon}\right\|_{L^{p}} \leq\left\|v_{\epsilon}\right\|_{Y} \leq c_{2},
$$

and from $\int_{0}^{k T} \tilde{u}_{\epsilon}(t) d t=0$, i.e.

$$
\left|\tilde{u}_{\epsilon}(t)\right| \leq\left|\int_{0}^{k T} \dot{\tilde{u}}_{\epsilon}(t) d t\right| \leq(k T)^{1 / q}\left\|\dot{\tilde{u}}_{1 \epsilon}\right\|_{L^{p}}+(k T)^{1 / p}\left\|\dot{\tilde{u}}_{2 \epsilon}\right\|_{L^{q}},
$$

we have $\left\|\tilde{u}_{\epsilon}\right\|_{X} \leq c_{3}$.

Meanwhile, by the convexity of $F(t, \cdot)$, we have

$$
\begin{aligned}
H(t, & \left.\bar{u}_{\epsilon} / 2\right) \\
& \leq \frac{1}{2} H\left(t, u_{\epsilon}(t)\right)+\frac{1}{2} H\left(t,-\tilde{u}_{\epsilon}(t)\right) \\
& \leq \frac{1}{2} H\left(t, u_{\epsilon}(t)\right)-\frac{1}{2} H(t, 0)+\frac{1}{2} H(t, 0)+\frac{\alpha}{2 p}\left|\tilde{u}_{1 \epsilon}(t)\right|^{p}+\frac{1}{2 \alpha} \gamma(t)+\frac{\alpha^{q-1}}{2 q}\left|\tilde{u}_{2 \epsilon}(t)\right|^{q} \\
& \leq \frac{1}{2}\left(\nabla H\left(t, u_{\epsilon}(t)\right), u_{\epsilon}(t)\right)+\frac{\alpha}{2 p}\left|\tilde{u}_{1 \epsilon}(t)\right|^{p}+\frac{\alpha^{q-1}}{2 q}\left|\tilde{u}_{2 \epsilon}(t)\right|^{q}+\frac{1}{\alpha} \gamma(t) \\
& =\frac{1}{2}\left(-J \dot{u}_{\epsilon}(t), u_{\epsilon}(t)\right)-\epsilon\left(\Phi_{p}\left(u_{1 \epsilon}(t)\right), u_{1 \epsilon}(t)\right)+\frac{\alpha}{2 p}\left|\tilde{u}_{1 \epsilon}(t)\right|^{p}+\frac{\alpha^{q-1}}{2 q}\left|\tilde{u}_{2 \epsilon}(t)\right|^{q}+\frac{1}{\alpha} \gamma(t) .
\end{aligned}
$$

So,

$$
\begin{aligned}
& \int_{0}^{k T} F\left(t, \bar{u}_{\epsilon} / 2\right) d t \\
& \quad \leq \int_{0}^{k T} \frac{1}{2}\left(-J \dot{u}_{\epsilon}(t), u_{\epsilon}(t)\right) d t+\frac{\alpha}{2 p}\left\|\tilde{u}_{\epsilon}\right\|_{L^{p}}^{p}+\frac{\alpha^{q-1}}{2 q}\left\|\tilde{u}_{2 \epsilon}\right\|_{L^{q}}^{q}+\gamma_{0} \\
& \quad \leq \frac{1}{2}\left\|\tilde{u}_{1 \epsilon}\right\|_{L^{p}}\left\|\dot{\tilde{u}}_{2 \epsilon}\right\|_{L^{q}}+\frac{1}{2}\left\|\dot{\tilde{u}}_{1 \epsilon}\right\|_{L^{p}}\left\|\tilde{u}_{2 \epsilon}\right\|_{L^{q}}+\frac{\alpha}{2 p}\left\|\tilde{u}_{\epsilon}\right\|_{L^{p}}^{p}+\frac{\alpha^{q-1}}{2 q}\left\|\tilde{u}_{2 \epsilon}\right\|_{L^{q}}^{q}+\gamma_{0} \\
& \quad \leq c_{4} .
\end{aligned}
$$

This together with condition $\left(\mathrm{A}_{3}\right)$ implies that $\left|\bar{u}_{\epsilon}\right|$ is bounded. Consequently,

$$
\left\|u_{\epsilon}\right\|_{X} \leq\left\|\bar{u}_{\epsilon}\right\|_{X}+\left\|\tilde{u}_{\epsilon}\right\|_{X} \leq c_{5} .
$$


Step 3: Existence of a solution for the problem (4).

Since $\left\|u_{\epsilon}\right\|_{X}$ is bounded, there is a sequence $\epsilon_{n} \rightarrow 0(n \rightarrow \infty)$ with $\epsilon_{n} \in\left(0, \epsilon_{0}\right)$ and a function $u \in X$ such that

$$
u_{\epsilon_{n}} \rightarrow u \quad \text { as } n \rightarrow \infty
$$

Moreover, from $\dot{v}_{\epsilon}=-J \dot{u}_{\epsilon}$, we have

$$
v_{\epsilon}(t)=-J\left(u_{\epsilon}(t)-\bar{u}_{\epsilon}\right) .
$$

So $\left\{v_{\epsilon_{n}}\right\}$ converges weakly to $v(t)=-J(u(t)-\bar{u})$. From (12), we have the integrated form

$$
\left\{\begin{array}{l}
u_{1 \epsilon_{n}}(t)-u_{1 \epsilon_{n}}(0)-\int_{0}^{t} \Phi_{q}\left(\alpha u_{2 \epsilon_{n}}(s)\right) d s=0 \\
u_{2 \epsilon_{n}}(t)-u_{2 \epsilon_{n}}(0)+\epsilon_{n} \int_{0}^{t} \Phi_{p}\left(\alpha u_{1 \epsilon_{n}}(s)\right) d s+\int_{0}^{t} \frac{1}{\alpha} \nabla F\left(s, u_{1 \epsilon_{n}}(s)\right) d s=0
\end{array}\right.
$$

Because $u_{\epsilon_{n}}$ converges weakly to $u$ in $X, u_{\epsilon_{n}}$ converges uniformly to $u$ in $C_{k T}^{\infty}$ (see Proposition 1.2 in [2]). So, let $n \rightarrow \infty$ and we have

$$
\left\{\begin{array}{l}
u_{1}(t)-u_{1}(0)-\int_{0}^{t} \Phi_{q}\left(\alpha u_{2}(s)\right) d s=0, \\
u_{2}(t)-u_{2 \epsilon_{n}}(0)+\frac{1}{\alpha} \int_{0}^{t} \nabla F\left(s, u_{1}(s)\right) d s=0
\end{array}\right.
$$

that is, $u$ is a solution of (4) in $X$.

Finally, we show $v=-J(u(t)-\bar{u})$ minimizes the dual action $\mathcal{X}$ on $\tilde{Y}$. Because $v_{\epsilon_{n}}$ is a minimum of $\mathcal{X}_{\epsilon_{n}}$ and $H_{\epsilon_{n}}^{*}(t, v) \leq H^{*}(t, v)$, we have

$$
\mathcal{X}_{\epsilon_{n}}\left(v_{\epsilon_{n}}\right) \leq \mathcal{X}_{\epsilon_{n}}(h) \leq \mathcal{X}(h), \quad \forall h \in \tilde{Y}
$$

By the duality between $u_{\epsilon_{n}}$ and $\dot{v}_{\epsilon_{n}}$ and the definition of $H_{\epsilon_{n}}(t, v)$, we have

$$
\begin{aligned}
\mathcal{X}_{\epsilon_{n}}\left(v_{\epsilon_{n}}\right) & =\int_{0}^{k T}\left(\frac{1}{2}\left(J \dot{v}_{\epsilon_{n}}(s), v_{\epsilon_{n}}(s)\right)+\left(\dot{v}_{\epsilon_{n}}(s), u_{\epsilon_{n}}(s)\right)-H_{\epsilon_{n}}\left(s, u_{\epsilon_{n}}(s)\right)\right) d s \\
& =\int_{0}^{k T}\left(\frac{1}{2}\left(J \dot{v}_{\epsilon_{n}}(s), v_{\epsilon_{n}}(s)\right)+\left(u_{\epsilon_{n}}(s), \dot{v}_{\epsilon_{n}}(s)\right)-H\left(s, u_{\epsilon_{n}}(s)\right)-\frac{\epsilon_{n}}{p}\left|u_{1 \epsilon_{n}}(s)\right|^{p}\right) d s .
\end{aligned}
$$

Moreover, as $v_{\epsilon_{n}}$ converges weakly to $v$ in $\tilde{Y}, J \dot{v}_{\epsilon_{n}}$ converges weakly to $J v$. Letting $n \rightarrow \infty$ we obtain, by $\dot{v}(t)=\nabla H(t, u(t))$,

$$
\begin{aligned}
& \lim _{n \rightarrow \infty} \mathcal{X}_{\epsilon_{n}}\left(v_{\epsilon_{n}}\right) \\
& =\lim _{n \rightarrow \infty} \int_{0}^{T}\left(\frac{1}{2}\left(J \dot{v}_{\epsilon_{n}}(s), v_{\epsilon_{n}}(s)\right)+\left(u_{\epsilon_{n}}(s), \dot{v}_{\epsilon_{n}}(s)\right)-H\left(s, u_{\epsilon_{n}}(s)\right)-\frac{\epsilon_{n}}{p}\left|u_{1 \epsilon_{n}}(s)\right|^{p}\right) d s \\
& =\int_{0}^{T}\left(\frac{1}{2}(J \dot{v}(s), v(s))+H^{*}(s, \dot{v}(s))\right) d s=\mathcal{X}(v) .
\end{aligned}
$$

So $\mathcal{X}(v) \leq \mathcal{X}(h)$ for all $h \in \tilde{Y}$. The proof is complete. 
Remark 3.1 Theorem 3.1 still holds if condition $\left(A_{1}\right)$ is changed to:

$\left(\mathrm{A}_{4}\right)$ There exists $l \in L^{p q /(p-m+1)}\left(0, k T ; \mathbb{R}^{N}\right)$ such that for all $x \in \mathbb{R}^{N}$ and a.e. $t \in[0, k T]$, one has

$$
F(t, x) \geq\left(l(t), \Phi_{m}(x)\right)
$$

where $m$ is an integer such that $2 \leq m \leq p$.

When the parameter $\alpha$ is smaller, we can obtain the prior bound for all the solutions of the $p$-Laplacian system (1).

Theorem 3.2 If there exist $\alpha \in\left(0, \min \left\{(2 k T)^{-p / q}, p(2 k T)^{-1}\right\}\right), \beta \geq 0, \gamma \geq 0$, and $\delta>0$ such that

$$
\delta|x|-\beta \leq F(t, x) \leq \frac{\alpha^{2}}{p}|x|^{p}+\gamma
$$

for a.e. $t \in[0, k T]$ and $x \in \mathbb{R}^{N}$, then each solution of (1) satisfies the inequalities

$$
\begin{aligned}
& \int_{0}^{k T}|\dot{x}(t)|^{p} d t \leq \frac{p k T(\beta+\gamma)}{p-2 k T \alpha}, \\
& \int_{0}^{k T}|x(t)| d t \leq \frac{k T(\beta+\gamma)}{\delta\left(1-2 k T \alpha^{q / p}\right)} .
\end{aligned}
$$

Proof We still set $u_{1}=x, \alpha u_{2}=\Phi_{p}(\dot{x})$, and the equalities (3) hold. Easily we find

$$
\frac{1}{q} \alpha^{-\frac{2 q}{p}}\left|\nabla F\left(t, u_{1}\right)\right|^{q} \leq\left(\nabla F\left(t, u_{1}\right), u_{1}\right)+\beta+\gamma
$$

It follows from

$$
(\nabla H(t, u), u)=\frac{1}{\alpha}\left(\nabla F\left(t, u_{1}\right), u_{1}\right)+\left(\Phi_{q}\left(\alpha u_{2}\right), u_{2}\right)
$$

that

$$
\frac{1}{q} \alpha^{-1-\frac{2 q}{p}}\left|\nabla F\left(t, u_{1}\right)\right|^{q}+\left(\Phi_{q}\left(\alpha u_{2}\right), u_{2}\right) \leq(\nabla H(t, u), u)+\frac{\beta}{\alpha}+\frac{\gamma}{\alpha} .
$$

That is,

$$
\frac{1}{q} \alpha^{-\frac{q}{p}}\left|\dot{u}_{2}(t)\right|^{q}+\alpha^{q-1}\left|u_{2}\right|^{q} \leq(-J \dot{u}(t), u(t))+\frac{\beta}{\alpha}+\frac{\gamma}{\alpha} .
$$

Integrating over $[0, k T]$ and using Lemma 2.6, we have

$$
\begin{aligned}
& \frac{1}{q} \alpha^{-\frac{q}{p}}\left\|\dot{u}_{2}\right\|_{L^{q}}^{q}+\alpha^{q-1}\left\|u_{2}\right\|_{L^{q}}^{q} \\
& \quad \leq-\int_{0}^{k T}(J \dot{u}(t), u(t)) d t+\frac{k T(\beta+\gamma)}{\alpha}
\end{aligned}
$$




$$
\begin{aligned}
& \leq \frac{2 k T}{p}\left\|\dot{u}_{1}\right\|_{L^{p}}^{p}+\frac{2 k T}{q}\left\|\dot{u}_{2}\right\|_{L^{q}}^{q}+\frac{k T(\beta+\gamma)}{\alpha} \\
& =\frac{2 k T}{p} \alpha^{q}\left\|u_{2}\right\|_{L^{q}}^{q}+\frac{2 k T}{q}\left\|\dot{u}_{2}\right\|_{L^{q}}^{q}+\frac{k T(\beta+\gamma)}{\alpha} .
\end{aligned}
$$

So,

$$
\left(\frac{1}{q} \alpha^{-\frac{q}{p}}-\frac{2 k T}{q}\right)\left\|\dot{u}_{2}\right\|_{L^{q}}^{q}+\left(\alpha^{q-1}-\frac{2 k T}{p} \alpha^{q}\right)\left\|u_{2}\right\|_{L^{q}}^{q} \leq \frac{k T(\beta+\gamma)}{\alpha}
$$

Hence

$$
\left\|u_{2}\right\|_{L^{q}}^{q} \leq \frac{p k T(\beta+\gamma)}{\alpha^{q}(p-2 k T \alpha)}, \quad\left\|\dot{u}_{2}\right\|_{L^{q}}^{q} \leq \frac{q k T(\beta+\gamma)}{\alpha^{1-q / p}-2 k T \alpha}
$$

and

$$
\left\|\dot{u}_{1}\right\|_{L^{p}}^{p}=\left\|\Phi_{q}\left(\alpha u_{2}\right)\right\|_{L^{p}}^{p}=\alpha^{q}\left\|u_{2}\right\|_{L^{q}}^{q} \leq \frac{p k T(\beta+\gamma)}{p-2 k T \alpha} .
$$

Meanwhile, by the convexity of $F$, we have

$$
\begin{aligned}
& \delta \int_{0}^{k T}\left|u_{1}(t)\right| d t-\beta k T \\
& \quad \leq \int_{0}^{k T} F\left(t, u_{1}(t)\right) d t \leq \int_{0}^{k T}\left(F(t, 0)+\left(\nabla F\left(t, u_{1}(t)\right), u_{1}(t)\right)\right) d t \\
& \quad \leq \gamma k T+\alpha \int_{0}^{k T}(\nabla H(t, u(t)), u(t)) d t-\alpha \int_{0}^{k T}\left(\Phi_{q}\left(\alpha u_{2}\right), u_{2}\right) d t \\
& \quad=\gamma k T-\alpha \int_{0}^{k T}(J \dot{u}(t), u(t)) d t-\alpha^{q}\left\|u_{2}\right\|_{L^{q}}^{q} \\
& \quad \leq \gamma k T+\alpha \frac{2 k T}{q}\left\|\dot{u}_{2}\right\|_{L^{q}}^{q} \leq \gamma k T+\frac{2 k^{2} T^{2}(\beta+\gamma)}{\alpha^{-q / p}-2 k T},
\end{aligned}
$$

which completes the proof.

\section{Subharmonic solutions}

Theorem 4.1 Assume that $F: \mathbb{R} \times \mathbb{R}^{N} \rightarrow \mathbb{R}$ is continuous. Suppose further that

$$
F(t, x) \rightarrow+\infty
$$

and

$$
\frac{F(t, x)}{|x|^{p}} \rightarrow 0
$$

as $|x| \rightarrow+\infty$ uniformly in $t \in \mathbb{R}$. Then for each $k \in \mathbb{R} \backslash\{0\}$, the system (1) has a $k T$-periodic solution $x_{k}$ such that

$$
\left\|x_{k}\right\|_{\infty} \rightarrow+\infty
$$

and such that the minimal period $T_{k}$ of $x_{k}$ tends to $\infty$ when $k \rightarrow+\infty$. 
Proof Let $c_{1}=\max _{t \in \mathbb{R}}|F(t, 0)|$. Then condition (16) implies that there exists a $r>0$ such that

$$
F(t, x) \geq 1+c_{1}
$$

for all $t \in \mathbb{R}$ and $x \in \mathbb{R}^{N}$ with $|x|>r$. By the convexity of $F$, we have

$$
\begin{aligned}
1+c_{1} & \leq F\left(t, \frac{r}{|x|} x\right) \leq \frac{r}{|x|} F(t, x)+\left(1-\frac{r}{|x|}\right) F(t, 0) \\
& \leq \frac{r}{|x|} F(t, x)+\left(1-\frac{r}{|x|}\right) c_{1},
\end{aligned}
$$

i.e.

$$
F(t, x) \geq \frac{1}{r}|x|+c_{1}
$$

for all $t \in \mathbb{R}$ and $x \in \mathbb{R}^{N}$ with $|x|>r$. Furthermore, the continuity of $F$ implies that there exist positive constants $\delta, \beta$ such that

$$
F(t, x) \geq \delta|x|-\beta
$$

for all $t \in \mathbb{R}$ and $x \in \mathbb{R}^{N}$.

By the condition (17), there exists $\alpha \in\left(0,(2 k T)^{-\max \{p, q\} / q}\right)$ such that

$$
F(t, x) \leq \frac{\alpha^{2}}{p}|x|^{p}+\gamma
$$

for all $t \in \mathbb{R}$ and $x \in \mathbb{R}^{N}$. Then from Theorem 3.1, we can see that the problem (4) has at least one solution $u_{k}=\left(u_{1 k}, u_{2 k}\right) \in X$ such that $u_{1 k}$ is the $k T$-periodic solution of (1) and

$$
v_{k}(t)=-J\left[u_{k}(t)-\frac{1}{k T} \int_{0}^{k T} u_{k}(s) d s\right]
$$

minimizes the dual action

$$
\mathcal{X}: \tilde{Y} \rightarrow(-\infty, \infty], \quad v \mapsto \int_{0}^{k T}\left(\frac{1}{2}(J \dot{v}(t), v(t))+H^{*}(t, \dot{v}(t))\right) d t
$$

Next we estimate the upper bound of $c_{k}=\mathcal{X}\left(v_{k}\right)$. For any $h=\left(h_{1}, h_{2}\right) \in \tilde{Y}$, we have

$$
\begin{aligned}
c_{k} & =\mathcal{X}\left(v_{k}\right) \leq \mathcal{X}(h)=\int_{0}^{k T}\left(\frac{1}{2}(J \dot{h}(t), h(t))+H^{*}(t, \dot{h}(t))\right) d t \\
& =\int_{0}^{k T}\left(\frac{1}{2}(J \dot{h}(t), h(t))+\frac{1}{\alpha} F^{*}\left(t, \dot{h}_{1}(t)\right)+\frac{1}{p \alpha}\left|\dot{h}_{2}\right|^{p}\right) d t .
\end{aligned}
$$

Let $\rho=\left(\rho_{1}, \rho_{2}\right) \in \mathbb{R}^{N} \times \mathbb{R}^{N}$ with $\left|\rho_{i}\right|=1, i=1,2$, and $\epsilon \leq 2 \pi \delta / k T$. Define the function

$$
h(t)=\frac{\epsilon k T}{2 \pi}\left(\rho \cos \frac{2 \pi}{k T} t+J \rho \sin \frac{2 \pi}{k T} t\right) .
$$


Obviously, $h \in \tilde{Y}$ and $J \dot{h}(t)=-\frac{2 \pi}{k T} h(t)$. Meanwhile, from (18), we can see that when $z \in \mathbb{R}^{N}$ with $|z| \leq \delta$, we have

$$
F^{*}(t, z) \leq \sup _{x \in R^{n}}((z, x)-F(t, x)) \leq \sup _{x \in R^{n}}((z, x)-\delta|x|+\beta) \leq \beta .
$$

So it follows from (20) that

$$
\begin{aligned}
c_{k} & \leq \mathcal{X}(h) \leq \int_{0}^{k T}\left(\frac{1}{2}(J \dot{h}(t), h(t))+\frac{\beta}{\alpha}+\frac{1}{p \alpha}\left|\dot{h}_{2}\right|^{p}\right) d t \\
& =-\frac{\epsilon^{2} T^{2}}{4 \pi} k^{2}+\left(\frac{\beta}{\alpha}+\frac{\epsilon^{p}}{p \alpha}\right) T k .
\end{aligned}
$$

If $\left\|x_{k}\right\|_{\infty} \rightarrow+\infty$ does not hold when $k \rightarrow+\infty$. Then there exist a subsequence $\left\{k_{n}\right\}$ and a constant $c_{1}>0$ such that

$$
\left\|x_{k_{n}}\right\|_{\infty} \leq c_{1}
$$

From (1), we have

$$
\left\|\frac{d}{d t} \Phi_{p}\left(\dot{x}_{k_{n}}\right)\right\|_{\infty} \leq c_{2}
$$

and so $\left\|\dot{x}_{k_{n}}\right\|_{\infty} \leq c_{3}$ for some constants $c_{2}, c_{3}>0$. This implies that

$$
\left\|v_{k_{n}}\right\| \leq c_{4}, \quad\left\|\dot{v}_{k_{n}}\right\| \leq c_{5}
$$

On the other hand, the inequality

$$
\begin{aligned}
c_{k_{n}} & =\mathcal{X}\left(v_{k_{n}}\right) \\
& =\int_{0}^{k_{n} T}\left(\frac{1}{2}\left(J \dot{v}_{k_{n}}(t), v_{k_{n}}(t)\right)+H^{*}\left(t, \dot{v}_{k_{n}}(t)\right)\right) d t \\
& \geq \int_{0}^{k_{n} T}\left(-\frac{1}{2}\left\|\dot{v}_{k_{n}}\right\|_{\infty}\left\|v_{k_{n}}\right\|_{\infty}-H(t, 0)\right) d t \\
& \geq-\left(\frac{1}{2} c_{4} c_{5}+\frac{\gamma}{\alpha}\right) T k_{n}
\end{aligned}
$$

is incompatible with (21) when $n$ is sufficiently large. Thus $\left\|x_{k}\right\|_{\infty} \rightarrow+\infty$ when $k \rightarrow+\infty$. It remains to prove that the minimal period $T_{k}$ of $x_{k}$ tends to $+\infty$ as $k \rightarrow+\infty$. If not, there exist $R>0$ and a subsequence $\left\{k_{n}\right\}$ such that the minimal period $T_{k_{n}}$ of $x_{k_{n}}$ is less than $R$. Meanwhile, by (18), (19), and Theorem 3.2, we have

$$
\begin{aligned}
& \int_{0}^{T_{k_{n}}}|\dot{x}(t)|^{p} d t \leq \alpha^{q}\left\|u_{2}\right\|_{L^{q}}^{q} \leq \frac{p T_{k_{n}}(\beta+\gamma)}{p-2 T_{k_{n}} \alpha}, \\
& \int_{0}^{T_{k_{n}}}|x(t)| d t \leq \frac{T_{k_{n}}(\beta+\gamma)}{\delta\left(1-2 T_{k_{n}} \alpha^{q / p}\right)} .
\end{aligned}
$$


We notice that

$$
\begin{aligned}
\left\|x_{k_{n}}\right\|_{\infty} & =\left\|\bar{x}_{k_{n}}+\tilde{x}_{k_{n}}\right\|_{\infty} \leq\left|\bar{x}_{k_{n}}\right|+\left\|\tilde{x}_{k_{n}}\right\|_{\infty} \\
& \leq \frac{1}{T_{k_{n}}} \int_{0}^{T_{k_{n}}}|x(t)| d t+T^{1 / q}\left(\int_{0}^{T_{k_{n}}}|\dot{x}(t)|^{p} d t\right)^{1 / p} .
\end{aligned}
$$

Inequalities (23) and (24) imply that $\left\{\left\|x_{k_{n}}\right\|_{\infty}\right\}$ is bounded, which is a contraction. The proof is complete.

\section{Competing interests}

The authors declare that they have no competing interests.

\section{Authors? contributions}

All authors contributed equally to the writing of this paper. All authors read and approved the final manuscript.

\section{Author details}

${ }^{1}$ School of Science, China University of Geosciences, Beijing, 100083, P.R. China. ${ }^{2}$ College of Mathematics and System Science, Shandong University of Science and Technology, Qingdao, 266590, P.R. China. ${ }^{3}$ Department of Mathematics, Texas A\&M University-Kingsville, Kingsville, TX 78363, USA.

\section{Acknowledgements}

This research is supported by the National Natural Science Foundation of China (No. 11101385) and by the Beijing Higher Education Young Elite Teacher Project.

Received: 28 August 2014 Accepted: 3 December 2014 Published online: 30 December 2014

\section{References}

1. Fonda, A, Ramos, M, Willem, M: Subharmonic solutions for second-order differential equations. Topol. Methods Nonlinear Anal. 1, 49-66 (1993)

2. Mawhin, J, Willem, M: Critical Point Theory and Hamiltonian Systems. Springer, Berlin (1989)

3. Rabinowitz, PH: On subharmonic solutions of Hamiltonian systems. Commun. Pure Appl. Math. 33, 609-633 (1980)

4. Tao, ZL, Tang, CL: Periodic and subharmonic solutions of second order Hamiltonian systems. J. Math. Anal. Appl. 293, 435-445 (2004)

5. Willem, M: Subharmonic oscillations of convex Hamiltonian systems. Nonlinear Anal. 9, 1303-1311 (1985)

6. Clarke, FH: Solution périodique des équations hamiltoniennes. C. R. Acad. Sci. Paris 287, 951-952 (1978)

7. Clarke, FH: A classical variational principle for periodic Hamiltonian trajectories. Proc. Am. Math. Soc. 76, 186-188 (1979)

8. Clarke, FH: Periodic solutions to Hamiltonian inclusions. J. Differ. Equ. 40, 1-6 (1981)

9. Clarke, FH, Ekeland, I: Nonlinear oscillations and boundary value problems for Hamiltonian systems. Arch. Ration. Mech. Anal. 78, 315-333 (1982)

10. Ekeland, I: Periodic solutions of Hamiltonian equation and a theorem of P. Rabinowitz. J. Differ. Equ. 34, 523-534 (1979)

11. Ekeland, I: Convexity Methods in Hamiltonian Mechanics. Springer, Berlin (1990)

12. Rockafellar, RT: Convex Analysis. Princeton Math. Series, vol. 28. Princeton University Press, Princeton (1970)

13. Mawhin, J, Willem, M: Critical points of convex pertubations of some indefinite quadratic forms and semi-linear boundary value problems at resonance. Ann. Inst. Henri Poincaré 3, 431-453 (1986)

14. Willem, M: Jean Mawhin?s contributions to critical point theory. Bound. Value Probl2013, 115 (2013). doi:10.1186/1687-2770-2013-115

15. Tian, Y, Ge, WG: Periodic solutions of non-autonomous second order system with a p-Laplacian. Nonlinear Anal. 66, 192-203 (2007)

16. Tian, Y, Graef, JR, Kong, LJ, Wang, M: Existence of solutions to a multi-point boundary value problem for a second order differential system via the dual least action principle. Discrete Contin. Dyn. Syst. 2013, suppl., $759-769$ (2013)

17. Lin, $X Y$, Tang, $X H$ : Existence of infinitely many solutions for $p$-Laplacian equations in $R^{N}$. Nonlinear Anal. 92, 72-81 (2013)

18. LV , X, Lu, SP, Yan, P: Periodic solutions of non-autonomous ordinary p-Laplacian system. J. Appl. Math. Comput. 35 11-18 (2011)

19. Ma, SW, Zhang, YX: Existence of infinitely many periodic solutions for ordinary p-Laplacian systems. J. Math. Anal. Appl. 351, 469-479 (2009)

20. Tang, XH, Zhang, XY: Periodic solutions for second-order Hamiltonian systems with a $p$-Laplacian. Ann. Univ. Mariae Curie-Skłodowska, Sect. A LXIV, 93-113 (2010)

21. Wang, ZY, Zhang, JH: Periodic solutions of non-autonomous second order systems with $p$-Laplacian. Electron. J. Differ. Equ. 2009, 17 (2009)

22. $\mathrm{Xu}, \mathrm{B}$, Tang, CL: Some existence results on periodic solutions of ordinary p-Laplacian systems. J. Math. Anal. Appl. 333 1228-1236 (2007)

23. Zhang, L, Chen, Y: Existence of periodic solutions of $p(t)$-Laplacian systems. Bull. Malays. Math. Soc. 35(1), 25-38 (2012) 
24. Zhang, XY, Tang, XH: Periodic solutions for second order Hamiltonian system with a $p$-Laplacian. Bull. Belg. Math. Soc. Simon Stevin 18, 301-309 (2011)

25. Manasevich, R, Mawhin, J: The spectrum of $p$-Laplacian systems under Dirichlet, Neumann and periodic boundary conditions. In: Morse Theory, Minimax Theory and Their Applications to Nonlinear Differential Equations. New Stud. Adv. Math., vol. 1, pp. 201-216. International Press, Somerville (2003)

doi:10.1186/s13661-014-0260-x

Cite this article as: Lian et al.: Periodic and subharmonic solutions for a class of second-order $p$-Laplacian Hamiltonian systems. Boundary Value Problems 2014 2014:260

Submit your manuscript to a SpringerOpen ${ }^{\circ}$ journal and benefit from:

- Convenient online submission

Rigorous peer review

- Immediate publication on acceptance

- Open access: articles freely available online

- High visibility within the field

- Retaining the copyright to your article

Submit your next manuscript at $>$ springeropen.com 